\title{
Age-related differences in diffusion model boundary optimality with both trial-limited and time-limited tasks
}

\author{
Jeffrey J. Starns • Roger Ratcliff
}

Published online: 6 December 2011

(C) Psychonomic Society, Inc. 2011

\begin{abstract}
In two-choice decision tasks, Starns and Ratcliff (Psychology and Aging 25: 377-390, 2010) showed that older adults are farther from the optimal speed-accuracy trade-off than young adults. They suggested that the age effect resulted from differences in task goals, with young participants focused on balancing speed and accuracy and older participants focused on minimizing errors. We compared speed-accuracy criteria with a standard procedure (blocks that had a fixed numbers of trials) to a condition in which blocks lasted a fixed amount of time and participants were instructed to get as many correct responses as possible within the time limit — a goal that explicitly required balancing speed and accuracy. Fits of the diffusion model showed that criteria differences persisted in the fixed-time condition, suggesting that age differences are not solely based on differences in task goals. Also, both groups produced more conservative criteria in difficult conditions when it would have been optimal to be more liberal.
\end{abstract}

Keywords Aging · Decision making $\cdot$ Response time models

Older adults are slower than young adults in many decision tasks (e.g., Baron \& Matilla, 1989; Rabbitt, 1979; Salthouse

\footnotetext{
J. J. Starns $(\square)$

Department of Psychology,

University of Massachusetts - Amherst,

441 Tobin Hall,

Amherst, MA 01003, USA

e-mail: jstarns@psych.umass.edu

R. Ratcliff

Department of Psychology, The Ohio State University,

1835 Neil Avenue,

Columbus, OH 43210, USA
}

\& Somberg, 1982), and a number of recent studies have explored this difference by applying the diffusion model (e.g., Ratcliff, Thapar, \& McKoon, 2003, 2004; Ratcliff, Thapar, Gomez, \& McKoon, 2004). This model assumes that evidence accumulates over time until it reaches one of two decision boundaries (e.g., "studied" versus "not studied" in a recognition memory task). The quality of the perceptual or mnemonic evidence driving a decision determines the rate with which the accumulation process approaches the correct boundary (the drift rate), but the process is subject to moment-to-moment variability that may result in termination on the incorrect boundary. The distance between the two decision boundaries determines the speed-accuracy trade-off: With narrow boundaries, the process terminates quickly but is likely to hit the wrong boundary because of within-trial variability. With wide boundaries, variability is less likely to lead to errors, but decisions are slow.

Diffusion model studies have found that aging has little or no effect on drift rates in many tasks (e.g., Ratcliff, Thapar, Gomez, et al., 2004; Ratcliff, Thapar, \& McKoon, 2001, 2004, 2010; Ratcliff et al. 2003), although drift rate differences have been observed in some contexts (Ratcliff, Thapar, \& McKoon, 2011; Thapar, Ratcliff, \& McKoon, 2003). Two other components of processing show much more consistent age differences: Older adults take longer at nondecision components of the task - such as reading a stimulus word or pressing a key once the decision has been made - and older adults set wider boundaries than younger adults. Thus, age-related slowing largely reflects different approaches to the speed-accuracy compromise, a conclusion that is bolstered by independent results in the aging literature (Smith \& Brewer, 1995; Rogers, Hertzog, \& Fisk, 2000; Touron, Hoyer, \& Cerella, 2004; White, Cerella, \& Hoyer, 2007). These results contrast starkly with models suggesting that aging leads to a global decrease in processing speed (e.g., Myerson, Hale, Wagstaff, Poon, \& Smith, 1990). 
Recently, Starns and Ratcliff (2010) used optimality analyses to explore why older adults consistently set wider boundaries than young adults. For a given decision task, a single boundary value leads to the most correct answers per unit time, a metric that is often referred to as reward rate (Bogacz, Brown, Moehlis, Holmes, \& Cohen, 2006; see Gold \& Shadlen, 2002 for application to the animal literature). Reward rate is only one of many possible ways to define an appropriate speed-accuracy trade-off, and the weighting of accuracy versus speed that is required in many ecological settings may be quite different than the weighting prescribed by reward rate optimality. For example, a student who correctly answers $90 \%$ of the questions on a test will get a better grade than a student who answers $80 \%$ correctly, even if the latter finishes in half the time (thus achieving a higher reward rate). Nevertheless, reward rate analyses can play a significant role in modeling how people set and adjust decision criteria - an important and largely unaddressed goal. Reward-rate optimal boundary (RROB) values provide an objective standard defining where the boundary would be placed if the participant was neither willing to respond substantially slower for relatively small gains in accuracy nor willing to become substantially less accurate for relatively small gains in speed (see Starns \& Ratcliff, 2010, Figs. 2 and 3). Therefore, RROB values provide an important reference point when considering how boundaries change across variables, populations, or tasks.

Starns and Ratcliff (2010) found RROB values for a number of data sets and compared them to the participants' actual boundary settings. For both young and older participants, actual boundary values were consistently wider than the RROB values, but younger participants were closer to rewardrate optimality than were older participants. Accuracy feedback and extensive task practice helped young participants more closely approximate RROB values, whereas these manipulations had no impact for older participants. Older adults almost never made avoidable errors; that is, their boundaries were so wide that their accuracy was near the maximum attainable given an infinite boundary width. Starns and Ratcliff attributed these results to age-based differences in task goals: Young participants attempted to balance speed and accuracy, whereas older participants attempted to minimize avoidable errors regardless of response speed.

The primary goal of the present experiment was to test the explanation for age differences offered by Starns and Ratcliff (2010) - that is, to determine whether age differences in boundary optimality simply reflect different task goals for young and older participants. We tested this account by including a condition in which both age groups were given the explicit goal of optimizing reward rate. In the Starns and Ratcliff experiments, participants completed blocks with set numbers of trials regardless of how long it took them to finish (we will call these fixed-trial blocks).
Thus, young and older participants were largely free to set their own goals regarding the relative importance of responding accurately versus finishing the blocks quickly, and responding slowly was the best strategy for getting the most correct answers. In the present experiment, we added a condition in which blocks lasted a set amount of time regardless of the number of trials completed (we will call these fixed-time blocks; see Balci et al., 2011; Bogacz, Hu, Holmes, \& Cohen, 2010; Simen et al., 2009). Fixed-time participants were told to get as many correct answers as possible within the time limit (that is, to maximize reward rate). Moreover, they were told that the number of errors did not matter, and that they may be able to get more correct responses by speeding up and completing more trials, even if this also led to more errors. If the only basis for the agerelated boundary difference is task goals, then the difference should be eliminated (or at least attenuated) when both groups are given the explicit goal of optimizing reward rate in the fixed-time condition.

In the present experiment, we included another deviation from the experiments analyzed by Starns and Ratcliff (2010) that allowed for more fine-grained optimality analyses. Starns and Ratcliff used existing diffusion model experiments that included a number of conditions differing in task difficulty, but these conditions were mixed within blocks. As a result, participants did not have an opportunity to change their boundaries across difficulty. In the present experiment, three difficulty conditions varied between blocks with the difficulty level signaled before each block began. Different boundary settings are required to optimize reward rate at different levels of difficulty (Balci et al., 2011; Bogacz et al., 2006). With extremely easy tasks, the correct answer can be produced even at a fast pace; thus, narrow boundaries are optimal. Extremely difficult tasks prioritize narrow boundaries for the opposite reason: Responding will be inaccurate even with a cautious pace, so there is little cost to fast responding. Between these extremes, wider boundaries are optimal to ensure that the available evidence leads to accurate responding. The present design allowed us to determine whether participants from either or both age groups matched the optimal pattern.

\section{Method}

\section{Participants}

The participants in the final data set included 18 older adults (nine each in the fixed-trial and fixed-time conditions) and 15 young adults (seven in the fixed-trial condition, eight in the fixed-time condition). All participants completed the Mini-Mental State Exam (MMSE; Folstein, Folstein, \& McHugh, 1975) as well as the vocabulary and matrix 
reasoning subtests of the Wechsler Adult Intelligence ScaleIII (Wechsler, 1997). Older adults were healthy individuals between the ages of 65 and 85 from a residential community in Columbus, $\mathrm{OH}$. To be included in the study, older adults had to score 26 or above on the MMSE and have no evidence of medical conditions causing cognitive impairments, head injuries with loss of consciousness, or a current psychiatric disorder. The final data set for older participants included 16 females and two males with the following average demographic scores: age $=74.4$; years of education $=$ 14.2; MMSE $=28.6$; vocabulary subtest $=46.1$ (11.6 scaled $)$; matrix reasoning subtest $=11.7 \quad(10.9$ scaled $)$; and $\mathrm{IQ}=107.3$. Young participants were college undergraduates at Ohio State University from the ages of 18 to 25 . Demographic data were unavailable for two participants in the young group. The remaining participants included eight females and five males, with the following average demographic scores: age $=20.5$; years of education $=13.0$; MMSE $=29.7$; vocabulary subtest $=45.9(12.5$ scaled $)$; matrix reasoning subtest $=21.1(13.3$ scaled $)$; and $\mathrm{IQ}=116.6$.

Six otherwise-eligible older adults were excluded from the data set. Four of these (three in the fixed-trial condition and one in the fixed-time condition) failed to follow instructions. One additional participant from the fixed-time condition appeared confused during the task, and another was excluded from the fixed-trial condition because of an extreme bias to use the "large" response. Two young participants (one in each condition) were excluded because of irregularities in their data. One showed chance performance on difficult blocks and had a high proportion of fast guesses (RTs below $250 \mathrm{~ms}$ ). The other had an outlier boundary value for the difficult condition (.154 with the other participants ranging from .074 to .116). We ensured that removing this participant from the fixed-time condition did not change any conclusions (including the effect of block type on boundary values). Removing the participant lowered the average boundary value on difficult tests by .006 and affected boundaries in the other difficulty conditions much less.

\section{Materials}

Participants completed the numerosity discrimination taska decision task that has been used to explore aging differences with the diffusion model (e.g., Ratcliff et al., 2001, 2010). For each trial, asterisks were displayed on the computer screen, and participants decided whether a "high" or "low" number was displayed. Stimuli were constructed by placing asterisks in a subset of spaces within a $10 \times 10$ pixel grid. The individual spaces to be filled with asterisks were randomly selected on each trial. Stimuli requiring a "high" response always displayed more than 50 asterisks, and stimuli requiring a "low" response always displayed fewer than 50 asterisks.
We constructed three sets of stimuli to create three levels of task difficulty. The number of asterisks in each stimulus was drawn from a uniform distribution with the following ranges by condition: easy high, 61-65; easy low, $35-39$; medium high, 56-60; medium low, 40-44; difficult high, 51-55; difficult low, 45-49.

\section{Design and procedure}

Participants completed three sessions. The first was considered a practice session and was excluded from data analyses. Each session comprised 30 blocks of the numerosity task. Participants were informed that they would see displays of varying numbers of asterisks, and for each display, they would decide whether there were under or over 50 asterisks. Participants made a "low" (under 50) response by pressing the " $\mathrm{z}$ " key and a "high" (over 50) response by pressing the " " key. Feedback followed each response; specifically, CORRECT or ERROR appeared on the screen for $300 \mathrm{~ms}$, followed by $100 \mathrm{~ms}$ of blank screen. Thus, the total interval between a response and the presentation of the next stimulus was $400 \mathrm{~ms}$, a relatively fast pace that makes response speed a significant factor in optimizing reward rate (Simen et al., 2009). Participants saw a TOO FAST message for any RT less than $250 \mathrm{~ms}$ to discourage responses that were not based on the information in the stimulus display, and this message remained on the screen for $600 \mathrm{~ms}$, followed by $300 \mathrm{~ms}$ of blank screen. In the fixed-trial condition, there were 40 trials in each block ( 20 with under 50 asterisks and 20 with over $50)$. In the fixed-time condition, blocks lasted for $30 \mathrm{~s}$, and each new trial had an equal probability of being under or over 50 asterisks. For both block conditions, there were 10 blocks in each of the three difficulty conditions, and the order of the difficulty conditions was randomized across blocks for each session. For the fixed-time condition, the total number of correct responses was displayed for $3 \mathrm{~s}$ following each block.

Participants in the fixed-trial condition were given standard instructions asking them to balance the speed and accuracy of their responding. Fixed-time participants were given the following instructions:

Each block of trials will last for 30 seconds, and you will keep getting new trials until time is up. Try to get as many correct answers as possible within each block. We are not concerned with the number of errors that you make - we only count your correct responses. To get the most correct answers, you should try to be both fast and accurate in your responding. If you are overly slow, you will not complete many trials before time is up, and you will have fewer opportunities to make correct responses. So, going faster may help you get more correct responses even if it also leads to more errors. 
The experimenter verified that each participant understood the instructions. Participants were reminded of the instructions at the beginning of the second session.

\section{Results}

RTs less than $250 \mathrm{~ms}$ and over 3,000 ms were excluded. The low cut-off eliminated $.3 \%$ of trials for young participants and less than $.1 \%$ for older participants. The high cut-off eliminated $.1 \%$ of trials for young participants and $.2 \%$ for older participants. The diffusion model was fit to each participant's data - that is, the response proportions and the $.1, .3, .5, .7$, and .9 quantiles of the RT distribution for correct and error responses in each of the difficulty conditions (cells with fewer than five responses were fit for accuracy but not RT quantiles). We used the $\chi^{2}$ fitting method (Ratcliff \& Tuerlinckx, 2002). The data had 66 total degrees of freedom. The diffusion model we applied had 17 parameters across the three difficulty levels. The critical parameters include the drift rate $(v)$, representing how quickly the evidence from the stimulus drives the accumulation process to the correct boundary; the boundary separation (a), representing the amount of accumulated evidence required to make a response; the starting point of evidence accumulation $(z)$, representing biases toward one or the other response before evidence arrives from the test stimulus; and nondecision time $\left(T_{e r}\right)$, representing the time needed for pre- and postdecisional processing (see Table 1 for a full list of the parameters with explanations for each). The average chi-squared value across all participants was 137 , with a range of 59-292. These results indicate an adequate fit to the data.

The average parameter values produced in the fits are reported in Table 1. The boundary results are analyzed in detail below. Here, we will briefly review the results for the nonboundary parameters. The starting point $(z)$ was consistently slightly above the midpoint of the boundary width, indicating a small bias to respond "high." As expected, drift rates $(v)$ decreased as difficulty increased, but they were similar across the age groups and block conditions. Nondecision time $\left(T_{e r}\right)$ was longer for older than for young participants.

For each participant, we found the RROB value in each difficulty condition with a search routine (see Starns \& Ratcliff, 2010). The routine adjusted the boundary width, whereas all other parameters were held at the values produced in fits to data (with starting point coded as a proportion of the boundary width). At each candidate

Table 1 Average parameter values from diffusion model fits to individual participants

\begin{tabular}{|c|c|c|c|c|c|c|c|c|c|}
\hline \multirow[t]{2}{*}{ Age, Block, and Difficulty } & \multicolumn{9}{|c|}{ Parameter } \\
\hline & $a$ & $z / a$ & $s_{Z}$ & $v$ & $d c$ & $\eta$ & $T_{e r}$ & $s_{t}$ & $p_{O}$ \\
\hline \multicolumn{10}{|l|}{ Young } \\
\hline \multicolumn{10}{|l|}{ Fixed-Trial } \\
\hline $\mathrm{E}$ & .105 & .523 & .037 & .400 & -.007 & .139 & 384 & 167 & .002 \\
\hline M & .114 & .517 & 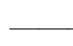 & .276 & -.004 & 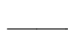 & 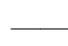 & - & \\
\hline $\mathrm{D}$ & .126 & .521 & 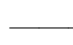 & .102 & -.009 & 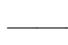 & 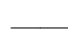 & 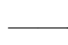 & 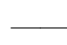 \\
\hline \multicolumn{10}{|l|}{ Fixed-Time } \\
\hline $\mathrm{E}$ & .077 & .512 & .029 & .370 & -.001 & .057 & 344 & 158 & .002 \\
\hline M & .086 & .518 & 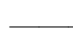 & .238 & -.003 & - & $\longrightarrow$ & - & \\
\hline $\mathrm{D}$ & .095 & .512 & 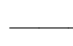 & .082 & -.013 & 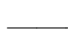 & 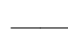 & 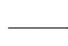 & 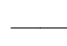 \\
\hline \multicolumn{10}{|l|}{ Older } \\
\hline \multicolumn{10}{|l|}{ Fixed-Trial } \\
\hline $\mathrm{E}$ & .132 & .513 & .017 & .395 & -.002 & .178 & 496 & 209 & .004 \\
\hline M & .149 & .513 & 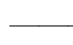 & .260 & -.006 & $\longrightarrow$ & 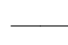 & $\longrightarrow$ & - \\
\hline $\mathrm{D}$ & .155 & .513 & 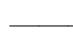 & .083 & -.021 & 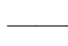 & 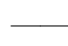 & 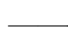 & 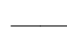 \\
\hline \multicolumn{10}{|l|}{ Fixed-Time } \\
\hline E & .097 & .546 & .028 & .364 & -.002 & .140 & 422 & 166 & .000 \\
\hline M & .114 & .546 & 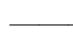 & .233 & -.005 & - & 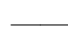 & 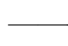 & 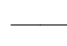 \\
\hline $\mathrm{D}$ & .123 & .546 & 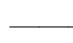 & .089 & -.014 & $\longrightarrow$ & 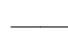 & 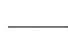 & 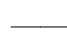 \\
\hline
\end{tabular}

Parameters in columns with marked-out cells were not free to vary across strength blocks, and the value in the "E" row was used for all three conditions. The remaining parameter columns report three freely varying parameters across the strength conditions. $E$ easy; $M$ medium; $D$ difficult. Parameter labels: $a$ = boundary separation; $z=$ starting point; $s_{Z}=$ range in starting point across trials; $v=$ mean drift rate (positive for over-50 stimuli, negative for under-50 stimuli); $d c=$ drift criterion (the zero-point in drift rate, so actual drifts are $v-d c$ ); $\eta=$ standard deviation in drift rates across trials; $T_{e r}=$ mean nondecision time; $s_{t}=$ range in nondecision time; $p_{O}=$ proportion of trials with RT contaminants 
boundary value, the routine evaluated the predicted accuracy and mean RT and used these values to calculate reward rate. This procedure was repeated until the boundary value producing the highest reward rate was found.

Figure 1 shows the RROB values, the participants' actual boundary values, and the deviation between the two. An ANOVA on the RROB values revealed that they did not differ for young (.077) and older (.073) participants, $F(1$, $29)=1.94, n s, M S E<.001$. RROB values also did not differ for fixed-trial blocks (.075) and fixed-time blocks (.075), $F(1,29)=1.94, n s$. However, RROB values did vary as a function of task difficulty, $F(2,58)=227.46, p<.001, M S E<$ .001 . To optimize reward rate, participants needed to use more narrow boundaries in the difficult condition (.057) than in either the medium (.082) or easy (.085) conditions. None of the possible interactions approached significance (lowest $p=.251$ ).

An ANOVA on participants' actual boundary values showed that older participants (.128) used wider boundaries than young participants $(.100), F(1,29)=17.52, p<.001$, $M S E=.001$. Boundary settings were also wider for fixedtrial blocks (.130) than for fixed-time blocks $(.099), F(1,29)=$ $22.66, p<.001$. In contrast with the pattern prescribed by reward rate optimality, participants used wider boundaries as difficulty increased (easy $=.103$, medium $=.116$, difficult $=$ $.125), F(2,58)=27.09, p<.001, M S E<.001$. No interactions were significant (lowest $p=.428$ ).

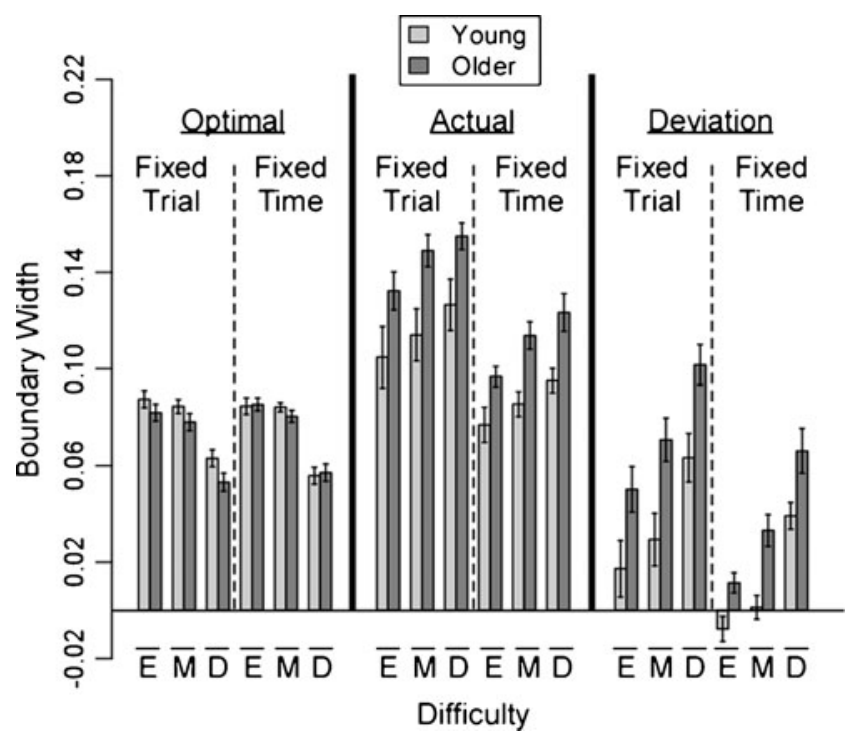

Fig. 1 Average boundary values for young and older adults (light and dark bars, respectively). The major divisions separate the reward-rate optimal boundary (RROB) values, the actual boundary values, and the deviation between the two (actual - RROB). Within each of these panels, the subpanel on the left shows results from fixed-trial blocks, and the subpanel on the right shows results from fixed-time blocks. Each pair of bars comes from one of the difficulty conditions ( $E$ easy; $M$ medium; $D$ difficult). The error bars show one standard error above and below each mean
Our primary interest was the deviation from the RROB value, which is shown in the rightmost panel of Fig. 1. Replicating previous work, actual boundary values were typically wider than the reward-rate optimal setting (shown as positive deviation scores). Older participants used boundaries that were farther from reward-rate optimal (deviation $=$ $.056)$ than those of young participants (deviation $=.022$ ), $F(1,29)=18.72, p<.001, M S E=.001$. Both age groups were closer to reward-rate optimal with fixed-time blocks $($ deviation $=.024)$ than with fixed-trial blocks (deviation $=$ $.053), F(1,29)=14.22, p=.001$. Indeed, the young participants in the fixed-time condition had a deviation score mean that was within one standard error of zero in the medium difficulty condition, and they actually used boundaries that were slightly more narrow than the RROB value in the easy condition [although the deviation was not significant, $t(7)=-1.48, p=.18]$. Although young participants were quite close to optimal in the easy and medium conditions, they did show as increase in boundary width from easy to medium that did not match the RROB values. Older participants remained more cautious than young participants in the fixed-time blocks, and the boundaries for older participants were significantly wider than RROB values even in the easy condition, $t(8)=2.74, p<.05$. Deviations from reward-rate optimality also varied across difficulty, $F(2,58)=$ $155.65, p<.001, M S E<.001$. Participants were farther from reward-rate optimality in more difficult conditions (easy = .017 , medium $=.033$, difficult $=.067$ ). None of the possible interactions were significant (lowest $p=.175$ ).

\section{Discussion}

Older participants used boundaries that were farther above the RROB value than those of young participants. Both groups were closer to reward-rate optimal in the fixed-time than in the fixed-trial condition. With fixed-time blocks, young participants used boundaries that were very close the RROB value in the medium difficulty condition, but their actual boundaries were slightly below the RROB value in the easy condition and well above this value in the difficult condition. Even with fixed-time blocks, older participants used actual boundaries that were wider than the RROB value in all difficulty conditions, although they were close to reward-rate optimal in the easy condition.

Most critically, the use of fixed-time blocks did not attenuate the age-related boundary differences. This suggests that the age differences cannot be wholly explained in terms of differences in task goals; that is, the explanation offered by Starns and Ratcliff (2010) is incomplete. The fixed-time paradigm confronts participants with a welldefined goal - get the most correct possible before the time limit - and reward rate analyses allow the experimenter to 
compute the exact speed-accuracy tradeoff needed to meet that goal. By comparing the actual and optimal boundaries with fixed-time blocks, we were able to measure the ability to adapt the speed-accuracy trade-off to an externally defined performance objective. The results suggest that young participants are better able to make targeted boundary adjustments than are older participants.

One difficulty in interpreting the reported results is that older participants might have simply chosen not to follow instructions on the fixed-time blocks as opposed to being unable to optimize reward rate. In this case, our results would indicate that older adults are more likely to disregard externally defined goals than are young participants. Decisively discriminating the "can't" and "don't want to" accounts will be difficult; however, our results do provide provisional support that older participants are less able to make targeted boundary adjustments. First, the older participants did respond substantially more quickly with fixed-time blocks than with fixed-trial blocks, demonstrating that they made some attempt to follow instructions. Many previous studies show that older participants also change boundaries in response to speed versus accuracy emphasis instructions, providing more evidence that older adults are willing to comply with experimenter-defined goals (Ratcliff, et al., 2001, 2003, Ratcliff, Thapar, \& McKoon, 2004; Thapar et al., 2003). Second, even the young participants showed limitations in their ability to optimize reward rate across the difficulty conditions with fixed-time blocks, and it is reasonable to think that these limitations would be more severe for the older population. Older participants might be less likely to explore different boundary settings or less able to monitor the accuracy and duration of their responses (Balci et al., 2011; Starns \& Ratcliff, 2010). The deficit might also have a physiological basis. For example, Forstmann et al. (2010) reported evidence that flexible speed-accuracy control is associated with strong connections between the presupplementary motor area and the striatum, and this circuit might be involved in aging effects as well.

As mentioned, neither age group optimized reward rate across all of the difficulty conditions. Specifically, participants in both age groups became increasingly conservative as task difficulty increased, whereas they should have been the least conservative in the most difficult condition. These results suggest that becoming more cautious when evidence becomes more ambiguous is a powerful heuristic, one that is intuitive and probably reinforced by everyday experience. Indeed, Balci et al. (2011) found that even young participants with $13-15$ sessions of practice on a decision task were too cautious to optimize reward rate in the difficult condition, despite performing close to reward-rate optimal in easier conditions.

Hawkins, Brown, Steyvers, and Wagenmakers (in press) reported evidence that participants respond as quickly as possible under the constraint that they stay above a minimum acceptable level of accuracy. Our difficulty results are consistent with the idea that participants attempt to maintain a target accuracy level (requiring wider boundaries in more difficult conditions). Both the minimum-accuracy strategy and reward-rate optimization will likely prove to be useful concepts in understanding speed-accuracy trade-offs. The minimum-accuracy strategy has the advantage of being easy to apply, making it a useful heuristic in explaining boundary setting in normal circumstances. However, it also has the disadvantage of leaving the acceptable minimum accuracy as a free parameter of the model. For example, if older participants set wider boundaries because they demand a higher minimum accuracy level, then we are still left with the question of why aging changes the acceptable accuracy level. Reward rate has the advantage of specifying a "parameter-free" target for an appropriate boundary value. Even if participants do not always attempt to optimize reward rate in normal circumstances, tasks like our own make reward rate optimization an explicit goal and offer an objective standard for success in meeting this goal (Balci et al., 2011; Bogacz et al., 2006; Bogacz et al., 2010; Gold \& Shadlen, 2002; Simen et al., 2009). As such, reward rate is an important tool in advancing our understanding of how participants control speed-accuracy criteria.

None of the current accounts of criteria setting address a critical difference between laboratory tasks and ecological decisions: Decision making outside the laboratory often involves only a single chance or relatively few chances to make a given decision. Thus, decision makers rarely have the experience they need to optimize reward rate or to minimize decision time for a target accuracy level. For situations such as these, theories must address how criteria settings are influenced by past experience in similar decision situations and by higher-level goals. For example, participants use different criteria when verbal instructions focus on speed versus accuracy, showing that criteria can be affected by goals that are defined very broadly. Moreover, participantlevel factors seem to play a large role, given that participants maintain fairly consistent criteria across a range of different decision tasks (Ratcliff, Thapar, \& McKoon, 2006; Ratcliff, et al., 2010, 2011). Thus, a complete account of speedaccuracy settings must consider a broad range of influences and is likely to remain a challenge for quite some time.

\section{Conclusion}

In comparison with standard fixed-trial paradigms with only vaguely defined goals regarding speed-accuracy trade-offs, the present results offer a more complete picture of age differences in boundary settings. Even when both age groups were given an explicit performance goal that 
required balancing speed and accuracy, age differences persisted. Moreover, both old and young participants had a limited ability to optimize reward rate across changing levels of difficulty. Overall, the results of the present experiment show that a complete account of age differences in speedaccuracy trade-offs must address not only task goals, but also the ability to achieve those goals.

Author Note Preparation of this article was supported by NIA Grant RO1-AG17083 and NIMH Grant R37-MH44640.

\section{References}

Balci, F., Simen, P., Niyogi, R., Saxe, A., Hughes, J., Holmes, P., \& Cohen, J. D. (2011). Acquisition of decision making criteria: Reward rate ultimately beats accuracy. Attention, Perception, \& Psychophysics, 73, 640-657.

Baron, A., \& Matilla, W. R. (1989). Response-slowing of older adults: Effects of time-limit contingencies on single- and dual-task performances. Psychology and Aging, 4, 66-72.

Bogacz, R., Brown, E., Moehlis, J., Holmes, P., \& Cohen, J. D. (2006). The physics of optimal decision making: A formal analysis of models of performance in two-alternative forced-choice tasks. Psychological Review, 113, 700-765.

Bogacz, R., Hu, P. T., Holmes, P., \& Cohen, J. D. (2010). Do humans produce the speed-accuracy tradeoff that maximizes reward rate? Quarterly Journal of Experimental Psychology, 63, 863-891.

Folstein, M. F., Folstein, S. E., \& McHugh, P. R. (1975). Mini-mental state: A practical method for grading the cognitive state of patients for the clinician. Journal of Psychiatric Research, 12, 189-198.

Forstmann, B. U., Schäfer, A., Anwander, A., Neumann, J., Brown, S. D., Wagenmakers, E.-J., ... Turner, R. (2010). Cortico-striatal connections predict control over speed and accuracy in perceptual decision making. Proceedings of the National Academy of Science, 107, 15916-15920.

Gold, J. I., \& Shadlen, M. N. (2002). Banburismus and the brain: Decoding the relationship between sensory stimuli, decisions, and reward. Neuron, 36, 299-308.

Hawkins, G., Brown, S. D., Steyvers, M., \& Wagenmakers, E.-J. (in press). Context effects in multi-alternative decision making: Empirical data and a Bayesian model. Cognitive Science.

Myerson, J., Hale, S., Wagstaff, D., Poon, L. W., \& Smith, G. A. (1990). The information-loss model: A mathematical theory of age related cognitive slowing. Psychological Review, 97, 475-487.

Rabbitt, P. (1979). How old and young subjects monitor and control responses for accuracy and speed. British Journal of Psychology, $70,305-311$.
Ratcliff, R., Thapar, A., Gomez, P., \& McKoon, G. (2004). A diffusion model analysis of the effects of aging in the lexical-decision task. Psychology and Aging, 19, 278-289.

Ratcliff, R., Thapar, A., \& McKoon, G. (2001). The effects of aging on reaction time in a signal detection task. Psychology and Aging, 16, 323-341.

Ratcliff, R., Thapar, A., \& McKoon, G. (2003). A diffusion model analysis of the effects of aging on brightness discrimination. Perception \& Psychophysics, 65, 523-535.

Ratcliff, R., Thapar, A., \& McKoon, G. (2004). A diffusion model analysis of the effects of aging on recognition memory. Journal of Memory and Language, 50, 408-424.

Ratcliff, R., Thapar, A., \& McKoon, G. (2006). Aging, practice, and perceptual tasks: A diffusion model analysis. Psychology and Aging, 21, 353-371.

Ratcliff, R., Thapar, A., \& McKoon, G. (2010). Individual differences, aging, and IQ in two-choice tasks. Cognitive Psychology, 60, $127-157$.

Ratcliff, R., Thapar, A., \& McKoon, G. (2011). Effects of aging and IQ on item and associative memory. Journal of Experimental Psychology: General, 140, 464-487.

Ratcliff, R., \& Tuerlinckx, F. (2002). Estimating parameters of the diffusion model: Approaches to dealing with contaminant reaction times and parameter variability. Psychonomic Bulletin \& Review, 9, 438-481.

Rogers, W. A., Hertzog, C., \& Fisk, A. D. (2000). An individual differences analysis of ability and strategy influences: Agerelated differences in associative learning. Journal of Experimental Psychology: Learning, Memory, and Cognition, 26, 359-394.

Salthouse, T. A., \& Somberg, B. L. (1982). Isolating the age deficit in speeded performance. Journal of Gerontology, 37, 59-63.

Simen, P., Contreras, D., Buck, C., Hu, P., Holmes, P., \& Cohen, J. D. (2009). Reward-rate optimization in two-alternative decision making: Empirical tests of theoretical predictions. Journal of Experimental Psychology: Human Perception and Performance, $35,1865-1897$.

Smith, G. A., \& Brewer, N. (1995). Slowness and age: Speed-accuracy mechanisms. Psychology and Aging, 10, 238-247.

Starns, J. J., \& Ratcliff, R. (2010). The effects of aging on the speedaccuracy compromise: Boundary optimality in the diffusion model. Psychology and Aging, 25, 377-390.

Thapar, A., Ratcliff, R., \& McKoon, G. (2003). A diffusion model analysis of the effects of aging on letter discrimination. Psychology and Aging, 18, 415-429.

Touron, D. R., Hoyer, W. J., \& Cerella, J. (2004). Cognitive skill learning: Age-related differences in strategy shifts and speed of component operations. Psychology and Aging, 19, 565-580.

Wechsler, D. (1997). The Wechsler Adult Intelligence Scale-Third edition. San Antonio, TX: Psychological Corporation.

White, A. S., Cerella, J., \& Hoyer, W. J. (2007). Strategy transitions during cognitive skill learning in younger and older adults: Effects of interitem confusability. Memory \& Cognition, 35, 2106-2117. 